\title{
Assessing Repair in Multiple Sclerosis: Outcomes for Phase II Clinical Trials
}

\author{
Maria Pia Sormani ${ }^{1}$ (D) Matteo Pardini ${ }^{2,3}$
}

Published online: 10 July 2017

(C) The American Society for Experimental NeuroTherapeutics, Inc. 2017

\begin{abstract}
Multiple Sclerosis (MS) pathology is complex and includes inflammatory processes, neurodegeneration, and demyelination. While multiple drugs have been developed to tackle MS-related inflammation, to date there is scant evidence regarding which therapeutic approach, if any, could be used to reverse demyelination, foster tissue repair, and thus positively impact on chronic disability. Here, we reviewed the current structural and functional markers (magnetic resonance imaging, positron emission tomography, optical coherence tomography, and visual evoked potentials) which could be used in phase II clinical trials of new compounds aimed to foster tissue repair in MS. Magnetic transfer ratio recovery in newly formed lesions currently represents the most widely used biomarker of tissue repair in MS, even if other markers, such as optical coherence tomography and positron emission tomography hold great promise to complement magnetic transfer ratio in tissue repair clinical trials. Future studies are needed to better characterize the different possible biomarkers to study tissue repair in MS, especially regarding their pathological specificity, sensitivity to change, and their relationship with disease activity.
\end{abstract}

Electronic supplementary material The online version of this article (doi:10.1007/s13311-017-0558-3) contains supplementary material, which is available to authorized users.

Maria Pia Sormani

mariapia.sormani@unige.it

1 Biostatistics Unit, Department of Health Sciences, University of Genoa, Genoa, Italy

2 Department of Neuroscience, Rehabilitation, Ophthalmology, Genetics, and Maternal and Child Health, University of Genoa, Genoa, Italy

3 Policlinic San Martino-IST, Genoa, Italy
Keywords Multiple sclerosis $\cdot$ Neuroprotection · Remyelination $\cdot$ Repair $\cdot$ Clinical trials

\section{Introduction}

Multiple sclerosis (MS) represents the prototypical demyelinating disease of the central nervous system and a frequent cause of life-long disability in the general population. MS is pathologically heterogeneous and as well as focal white matter (WM) demyelination it is characterized by widespread neurodegenerative changes (i.e., neuroaxonal loss) and infiltration of inflammatory cells [1]. This pathological milieu is highly dynamic and these tissue changes are often associated with spontaneous focal partial remyelination and functional architecture reorganization, more so in the earliest phases of the disease.

The last decade has seen an impressive change in the therapeutic landscape of MS with $>15$ disease-modifying treatments (DMT) currently approved to treat MS. Despite their differences in mechanism of action, all these treatments mainly modulate the inflammatory component of MS neuropathology, as shown by their profound impact on some magnetic resonance imaging (MRI) metrics of inflammation such as the formation of new WM lesions or pathological gadolinium enhancement. Given the wealth of anti-inflammatory treatments currently available for MS, the focus of pharmacological research is slowly but surely moving toward the development of neuroprotective and reparative compounds. The aim of neuroprotection is to try to reduce the impact of the disease on neuroaxonal loss and to prevent secondary neurodegeneration, whereas reparative treatments aim to mend existing damage by leading to remyelination, restoration of lost synaptic connections, and, ideally, reversal of previous neuroaxonal loss, facilitating endogenous repair processes, or participating directly in tissue repair [2]. Among those 
mechanism of tissue repair, remyelination seems the more achievable in the near future (and, indeed, some phase I and II trials of remyelinating compounds have been completed or are underway), but it is not the only possible avenue for tissue repair.

The aim of this work is to review the available markers of tissue damage that could be used in phase II clinical trials to evaluate DMT with potentials to foster or impact on tissue repair in MS, mainly focusing on imaging techniques. We will try to underline not only the potential of each marker to probe tissue repair in experimental settings, but also their applicability to multicenter clinical trials.

\section{MRI Outcomes}

\section{Assessment of WM Lesions: Lesion Volume}

WM lesions are the pathological hallmark of MS and represent focal areas of demyelination, associated with variable degrees of inflammatory infiltrates, axonal loss, and edema [1]. WM lesions can occur anywhere in the central nervous system in MS; however, some regions such as the corpus callosum, the periventricular WM, and the cerebellar peduncles are frequently lesioned in this condition. From a pathological point of view, WM lesions are dynamic over time: acute MS lesions, for example, are heavily infiltrated by macrophages and are rich in myelin debris and patchy signs of remyelination, whereas chronic lesions are markedly demarked from the surrounding tissue, hypocellular, and devoid or remyelinating axons [1]. WM lesions thus represent a privileged site in which to study spontaneous tissue repair and potentially DMT with reparative abilities.

WM lesion load quantification represents the most commonly used MRI measure in MS clinical trials. Usually WM matter lesions are identified using T2-weighted or T1weighted imaging (henceforth referred to, respectively, as $\mathrm{T} 2 \mathrm{~W}$ lesions and $\mathrm{T} 1 \mathrm{~W}$ lesions). $\mathrm{T} 2 \mathrm{~W}$ lesions are not pathologically specific and represent myelin and axonal loss, remyelination, and edema [3] and thus a cross-sectional evaluation of $\mathrm{T} 2 \mathrm{~W}$ lesion is not a good marker of tissue repair.

T1W lesions are relatively hypointense compared with the surrounding WM tissue. As for T2W lesions, the quantification of T1W lesion load per se is probably a poor marker of tissue repair as a reduction of $\mathrm{T} 1$ intensity is thought to represent a local expansion of extracellular space due to edema or to tissue destruction [4]. Moreover, while changes in myelin content can impact on T1 intensity, especially in newly formed lesions [5], pathological studies suggested that in chronic MS lesions T1 hypointensity mainly represented the extent of axonal loss [6].

Longitudinal studies of lesion evolution, however, could represent a more useful conventional MRI-based marker of tissue repair [7]. In a WM lesion life cycle, the first 6 months after its formation are the most interesting from a repair biomarker perspective. This time period can be divided in an "early" phase, lasting about a month and characterized by the disappearance of contrast enhancement and a "late" phase in which lesion volume and T1 and T2 intensities change [8]. Usually, volume of individual lesions, as assessed with T2weighted sequences is reduced in the "late" phase, and this is thought to represent both edema resolution (especially in the first weeks after the end of contrast enhancement) as well as noninflammatory processes such as degeneration and repair [9]. In the same time frame (i.e., 6 months from lesion formation), WM lesions can also present with changes in T1 intensity, with more than half of newly formed, acutely hypointense T1 lesions reverting to isointensity, possibly representing edema reduction, lower tissue damage, or partial remyelination [5]. The reduction of the formation of persistent T1hypointense lesions has been previously used in clinical trials but as an index of reduced acute tissue damage [10]; thus given that $\mathrm{T} 1$ lesions show poor specificity for myelin and are strongly influenced by neuroaxonal loss and edema, change in the rate of new $\mathrm{T} 1$ hypointesity formation is likely to be of only indirect value in remyelination and repair clinical trials.

\section{Assessment of WM Lesions: Magnetization Transfer Ratio}

Magnetization transfer ratio (MTR) is a semi-quantitative measure of the proportion of protons bound to macromolecular structures (such as lipids) relative to those that are in free water. In pathological studies, lesional MTR has been shown to strongly correlate with myelin density [11] and to be influenced by demyelization and axonal loss, as well as, to a minor extent, by inflammatory infiltrates and edema [12]. Remyelination has been shown to increase lesional MTR values, which usually remain lower than those observed in normal-appearing WM (NAWM) [11].

The changes in lesional MTR values over time are well described. Up to 3 months before the development of a new WM lesion, local reduction of MTR values can be observed [13], followed by a sharp decline in MTR values at the beginning of the gadolinium-enhancing phase, due to local edema, inflammation, and demyelination. In the following months, MTR partly or completely recovers due to reduction of edema and of spontaneous remyelination of remaining axons [14, 15]. Lesional MTR recovery represents an easy-to-measure approach to tissue repair in MS. Different approaches have been proposed to enhance the yield of lesional MTR recovery as a measure of tissue repair such as focusing only on newly formed lesions (as chronic lesions have a lower potential to undergo remyelination) and to separately analyze MTR changes over time on a lesion-by-lesion basis (to better 
account for baseline interlesional heterogeneity) [16]. MTR imaging is feasible in multicenter studies, even if care is needed to guarantee homogeneous protocol between centers given the dependency of MTR on the sequence parameters used.

Sample sizes have been calculated to use lesional MTR recovery as a marker of lesion repair in relapsing-remitting MS (RRMS), showing that $<70$ subjects per arm could be enough to identify at least $30 \%$ of MTR improvement in WM lesions [17-19].

Indeed, lesional MTR recovery has been already used in the past in clinical trials on injectable medications in MS, showing a speedier recovery of lesional MTR at the end of gadolinium enhancement period in treated versus nontreated subjects [14]. Moreover, lesional MTR has been already been used as a primary outcome measure in multicenter trials of putative remyelinating agents in MS [20] showing the clinical value and the technical maturity of this approach. To date lesional MTR changes over time thus represent one of the most promising measures to assess repair in MS [19].

Different groups are currently working on improving MTR to reduce its dependency on sequence parameters and increase its pathological specificity. A possibility is to acquire multiple images with different MT weighting to compute quantitative MT (qMT) (as opposed to the acquisition of only 2 images - 1 with MT weighting and 1 without-for MTR). qMT allows us to quantify different parameters such as the fraction of protons attached to macromolecules, which are reproducible and present good estimates of myelin loss [21]. Acquisition of qMT data, however, is time-consuming and the fitting of the underling mathematical model is not without challenges, thus currently limiting the use of qMT to the research setting.

\section{Brain and Spinal Cord Volume}

In MS, brain volume changes represent the final common pathway of a number of different pathological processes including not only neuroaxonal loss and demyelination, but also reduction of edema and of inflammation [22]. Patients with MS have been shown to present a more marked reduction of volume over time compared with healthy controls [22] and volume loss has been shown to significantly correlate with disability [23]. Different approaches have been proposed to quantify brain volume using clinically available, volumetric, lesion-filled T1 images, with good intercenter reproducibility [24], and in recent years fully automated, Food and Drug Administration-approved software packages have been developed to do so [25], potentially ushering the use of atrophy data in clinical practice in MS. Annualized atrophy rate has been included in the majority of recently designed trials for DMT in MS and more recently it has been included in a combined index of absence of disease activity together with absence of MRI activity (new T2 and/or gadolinium-enhanced T1 lesions), relapses and disability progression (i.e., the no evidence of disease activity-4 index) [26].

Reduction of annualized atrophy rate represent an ideal target for neuroprotection trials and sample size calculations for the use of normalized brain volume changes this setting are available [27, 28]. However, the use of brain atrophy-based measures to evaluate tissue repair is problematic, especially in a dynamic condition such as MS, where untangling the relative contribution of neuroprotective and reparative effects of a drug on these metrics loss could be difficult. Moreover, some processes playing a role in tissue repair such as reduction of inflammatory infiltrates and resolution of edema are associated with a reduction of brain volume (i.e., pseudoatrophy) [29], whereas others, such as remyelination could lead to an increase of brain volume.

Beside brain volume, the cross-sectional area of the cervical spinal cord has received interest as a possible biomarker of neurodegeneration in MS. Cervical cord atrophy is a frequent and early finding in MS and predicts disability independently from brain atrophy [30]. To date, cervical cord volume quantification is relatively less standardized than brain atrophy even if it has been included as a surrogate outcome measures in neuroprotective therapeutic trials [31]. Cervical cord atrophy usually requires dedicated imaging (i.e., volumetric cervical cord sequences), thus increasing the length of the MRI acquisition, even if quantification of the medulla oblongata volume [32] or of the lower brainstem cross-sectional area on volumetric brain imaging [33] have been shown to be possible easy-to-acquire proxy measures of cervical cord volume loss.

\section{Normal Appearing Tissue Imaging: Diffusion Weighted Imaging and MTR}

NAWM represent those WM regions free of visible discrete lesions. Two factors need to be taken into account when evaluating NAWM, that is, the dependence on MR magnet strength on the ability to identify WM lesions [34] and the presence of an association between proximity to lesions and tissue damage (i.e., NAWM perilesional tissue is usually more damaged than NAWM farter from lesions) [35]. Damage in NAWM, moreover, is not evenly distributed with regions nearer to the inner or outer surfaces of the brain presenting with more severe tissue abnormalities both in relapse-onset MS [36, 37] and in subjects with a clinically isolated syndrome [38]. Pathological studies showed the presence of diffuse microglia activation, gliosis, and variable extent of axonal loss in NAMW in MS. Axonal density is decreased in NAWM by $12 \%$ to $42 \%$, depending on the area studied, with the corpus callosum presenting with more intense pathology than other brain areas [39]. To date the relative contributions of wallerian degeneration due to lesional pathology and local 
NAWM damage in leading to NAWM neuroaxonal loss is unclear; however, the correlation between WM lesion load and NAWM damage seems weak at best [40].

In MS, NAWM has been usually studied with MTR and with diffusion imaging techniques. The considerations previously reported for lesional MTR are also valid for NAWM MTR. Local differences in NAWM MTR, moreover, are associated with different pathological substrata, with NAWM regions presenting with normal or slightly altered MTR values also showing less severe pathology compared with NAWM regions with lower MTR values [41]. Longitudinal studies, moreover, showed that regions bound to become WM lesions presented local NAWM MTR changes, as early as 3 months before lesion formation, suggesting the ability of MTR to capture prelesional changes in NAWM [13].

Diffusion weighted imaging evaluates the diffusion of water molecules along different directions to probe tissue integrity and its spatial architecture. Different mathematical models have been developed to interpret diffusion data. Among those the most widely used is diffusion tensor imaging (DTI), which requires the acquisition of diffusion-sensitized images along at least 6 directions to fit the model and compute the different metrics. The most widely used DTI measures are fractional anisotropy (FA), mean diffusivity (MD) and axial and radial diffusivity, representing, respectively, the presence of anisotropic diffusion (FA; i.e., the diffusion of water along a preferred direction), the overall diffusion of water (MD), and the diffusion along the primary diffusion direction (axial diffusivity) and along an axis perpendicular to it (radial diffusivity). In regions where WM fibers are mainly parallel one to the other (e.g., the corpus callosum), the interpretation of DTI indices is straightforward and can usefully inform on the underling pathological process with a reduction in FA and an increase in $\mathrm{MD}$, usually associated with tissue damage and change in radial diffusivity often associated with demyelination [42]. Among those measures, FA perhaps is the most widely reported index and in postmortem imaging studies of MS brains it has been shown to capture both axonal loss and demyelination [43], even if it is also sensible to the presence of local edema and to the local spatial tissue organization [44]. Axial and radial diffusivity are even more sensible than FA to local architecture and thus it is important to exercise caution in their pathological interpretation in those regions those rich in crossing WM fibers [45]. To try to cope with the difficulty in the pathological correlations of DTI findings in MS, other mathematical models have been developed. An example is the Neurite Orientation Dispersion and Density Imaging approach [46], which allows to differentiate the density of fibers (i.e., neurite density) from their spatial orientation (i.e., neurite dispersion), thus reducing the impact of local architecture differences on the interpretation of diffusion findings. Future studies, however, are needed to explore the applicability of neurite orientation dispersion and density imaging in multicenter studies.
From a clinical trial biomarker perspective, both NAWM MTR and diffusion data could represent useful metrics to explore tissue repair and approaches to reduce DTI and MTR intercenter variability in MS or in other neurological conditions have been proposed in different studies $[47,48]$. Indeed, in recent years, as for lesional MTR [20], also normalappearing tissue MTR has been successfully used in multicenter MS clinical trials [49].

\section{Positron Emission Tomography}

Positron emission tomography (PET) uses probes labeled with a radioactive isotope, which can bind to or be converted by a specific biological target, and thus allows to evaluate different functional or molecular facets of pathology. The PET tracer most commonly used in neurology is $2^{\prime}-[18 \mathrm{~F}]$ fluoro-2' deoxyglucose (18F-FDG), which allows to probe local brain metabolism. In MS, it has mainly been used to evaluate the metabolic activity of lesions (i.e., to divide lesions in active or chronic) even if the high background glucose uptake of the brain reduces the potential of $18 \mathrm{~F}-\mathrm{FDG}$ to image small lesions. Thus, $18 \mathrm{~F}-\mathrm{FDG}$ represents in MS mainly a marker of inflammation rater than of tissue repair [50].

Recent years have seen the development of a number of PET tracers for the identification of brain amyloid to aid in the diagnosis of Alzheimer's disease. The Pittsburg compound B (thioflavin-T derivative [methyl-11C]-2-(40methylaminophenyl)-6-hydroxybenzothiazole; 11C-PiB) represent the prototypical member of this class and has been shown to image not only amyloid, but also to present with diffuse WM binding, thought to represent an unspecific binding to myelin. In MS, $11 \mathrm{C}-\mathrm{PiB}$ has been shown to bind more strongly to NAWM rather than perilesional tissue and lesions, in line with the relative loss of myelin in these regions [51, 52]. Longitudinal data, moreover, showed the ability of 11C$\mathrm{PiB}$ to capture remyelination and demyelination in single lesions over time and, indeed, to this aim indices of lesional remyelination and demyelination have been proposed based on $\mathrm{PiB}$ intralesional uptake changes over time [52]. 11C-PiB, however, is not available commercially as given it short halflife needs to be synthetized right before the injection using dedicated cyclotron facilities, thus limiting its availability to a reduced number of centers. From a clinical trial perspective, the availability of fluorinated amyloid PET tracers (i.e., compound with a longer half-life compared to 11C-PiB), such as Florbetapir, Flutemetamol or Florbetaben, which are approved for clinical use by regulatory agencies, available commercially and already used in therapeutic trials of Alzheimer's disease, make the use of amyloid PET in tissue repair studies a realistic possibility. To this aim, however, more work is needed regarding the behavior of fluorinated amyloid PET tracers in MS [53]. Moreover, the higher cost of PET studies and the lower 
availability of PET imagers compared with MRI, are limiting factors in the acquisition of large PET datasets in MS.

\section{Functional MRI}

Functional MRI (fMRI) is based on the assessment of regional changes in blood oxygenation to determine the state of neuronal activation. fMRI data can be collected while the subject is performing a cognitive or motor task (i.e., task-based fMRI) usually to characterize the neural substrate of a specific brain function [54], or while the subject is at rest (i.e., resting-state fMRI) to shed light on the patterns of co-activation between different brain areas.

In MS, fMRI has been used to explore the impact of structural damage on the functional architecture of the brain. Overall, a relatively coherent pattern has been observed using task-related fMRI in MS, showing in the majority of studies an increased recruitment of brain areas compared to controls, possibly with an adaptive role to sustain performance and to contrast the impact of tissue pathology $[55,56]$. This increased of cortical recruitment associated with the severity of tissue pathology seems to follow a stereotyped pattern with a progressive involvement of those areas usually active in healthy controls during the task, followed by the recruitment of homotopic contralateral areas and lastly of nontask-specific prefrontal and deep gray matter areas [57]. Interestingly, this pattern of progressive recruitment has been observed in healthy controls with the increase of task complexity.

It must be noted, however, that not always the increase in brain activity presents with an adaptive value. In patients with progressive MS, the reduction of the activity in primary sensory-motor cortices associated with an increase in activity in associative higher-order areas has been related to reduced motor performance, possibly due to a switch from a taskspecific to a nontask-specific activation pattern [58]. Moreover, increased brain activity during a motor task has been associated with perceived fatigue, which could thus possibly represent the subjective correlate of the functional anatomy modification observed in MS [59,60]. Overall, it has been proposed that changes in the fMRI patterns toward the healthy controls functional anatomy could represent a marker of tissue repair; more studies, however are needed to reach a consensus on this issue [61].

The ability of fMRI-based techniques to capture tissue reorganization makes them an attractive biomarker for the study of tissue repair. Despite the wealth of data collected using fMRI both in healthy subjects and in patients with neurological conditions, different factors limit its usefulness in multicenter trials. A first problem is represented by the reproducibility of fMRI results in patients with MS. Different factors including changes in attention, fatigue, reduced sleep quality, and caffeine intake all significantly impact on fMRI patterns
[62]. Indeed, multicenter data seem to suggest that intrasubject reproducibility of fMRI data is lower in patients with MS patients - which frequently present the aforementioned confounding factors - than in controls [63]. A second problem is the difficulty in the interpretation of changes in fMRI patterns in the setting of a restorative therapy trial, that is, to disentangle the impact on fMRI results of tissue repair from those mediated by improved performance or reduced effort during the task.

Resting state fMRI, however, is free from the confounding effect of task performance (as it is task-free) even if it is prone to other possible confounding factors both in the acquisition (e.g., subject drowsiness) and the analysis (e.g., the lack of comparability of resting state fMRI results based on different cortical parcellation schemes) phases [64]. Despite this, resting-state fMRI has been successfully used to probe the impact on functional connectivity of motor and cognitive rehabilitation approaches in MS [65], and as a possible marker of treatment response in psychiatric conditions [66] and thus - pending the resolution of the methodological and interpretative problems - it could represent an useful approach to assess the downstream effect of tissue repair on functional anatomy.

\section{MRI Spectroscopy}

MRI spectroscopy (MRS) is used to study metabolites in the brain, as long as they present 1 or more MR visible nuclei. In MS, the most common approach is to use proton MR spectroscopy (1H-MRS), which allows us to quantify a number of metabolites, including $N$-acetyl aspartate (NAA), creatine, choline, and myoinositol [67]. NAA is the most widely used MRS marker and it is thought to be an index of neuroaxonal density [68]. NAA correlates with disability [69] and subclinical progression of atrophy [70]. The use of MRS, however, is problematic to assess tissue repair in clinical trials, among its main problems are the difficulty in assessing normal myelin, lack of whole brain coverage, long acquisition times, and the low spatial resolution [67]. Moving away from NAA, other MRS markers, such as myoinositol and glutamate, hold promise in terms of specificity. A recent study [71] on a large cohort $(n=$ 325) of patients with MS followed for 5 years, indicates that higher glutamate concentrations increase the rate of NAA decline, and higher glutamate/NAA ratio (in the NAWM) increases the rate of decline of brain volume, MSFC, and PASAT. This provides evidence of a relationship between brain glutamate and markers of disease progression in MS. Longitudinal studies are needed, however, to assess the value of these markers as outcome of clinical trials for repair. 


\section{Combined Measures}

Overall, none of the aforementioned measures is specific for a single pathological process. This is a key problem in MS, given the pathological heterogeneity underling this disorder and a possible cause of the clinico-radiological paradox observed in this population (i.e., the moderate correlations between MRI and disability measures) [72]. This is also problematic from a biomarker perspective as it reduces the certainty of the pathological process measured.

A possible solution is to try to combine different MRI measures in single indices thus trying to build on the strengths of different imaging modalities to increase the amount of the captured pathology. In a seminal study, for example Matthews et al. [73] showed that taking into account both lesional MRI spectroscopy and WM lesion volume alone better captured the differences in lesion pathology between patients with RRMS and secondary progressive MS.More recently, it has been shown that using a principal component analysis to combine volume, diffusion, and MTR measures in a single metrics a marked improvement on clinicoradiological correlations was obtained on the use of a single modality alone [74]. Interestingly, similar results have also been shown using zscores to quantify the composite indices, thus reducing the complexity of the previous approach [75]. The use of combined measures, however, is not without difficulties. First of all, the choice of which metrics to combine has been to date arbitrary. Moreover, while this approach increases the spectrum of the pathological abnormalities captured it also probably reduces the pathological specificity (i.e., it is a catch-all approach), which, as we acknowledged before, poses serious problems in the interpretation of therapeutic intervention. Lastly, the combination of multiple MRI measures presents with the risk of increasing the measurement errors, more so for these sequences not routinely performed in clinical trials. A possible solution, in our opinion, is represented by the pathological validation of these combined indices based on combined MRI-pathological studies, as has been previously done for different MRI approaches such as MTR or DTI [11, 41, 43]. It must be noted, however, that the use of composite MRI indices based on multiple sequences significantly increases the time needed to acquire each MRI scan, as well as the cost for each scan. These concerns, together with the reduced availability of some sequences in commercially available MRI scans, could reduce the applicability of these approaches to large studies.

\section{Visual System Measures: Optical Coherence Tomography and Visual Evoked Potentials}

The visual system is a major target of MS pathology and disability. Visual acuity measurements in patients with MS, through use of low-contrast sensitivity charts, show deficits that are related to the visual pathways [76], correlated to Expanded Disability Status Scale [76] and show response to treatments mirroring benefits on Expanded Disability Status Scale [76]. Therefore, visual acuity has been proposed as a clinical outcome to be included in phase III clinical trials, often in combination with other clinical measures [76].

The retina is composed of multiple layers of neurons and the innermost layer (the retinal nerve fiber layer (RNFL) is composed of unmyelinated axons that arise from the retinal ganglion cells and converge in the myelinated optic nerve [77]. The RNFL is unique in that it can be directly visualized through fundoscopic examination.

Optical coherence tomography (OCT) is an established technology, based on a noninvasive and inexpensive technique [77], that uses the reflection patterns of infrared light off the retinal layers to quantify the RNFL and other retinal structures such as the ganglion cell and inner plexiform (GCIP) layers. Specifically in MS, OCT could be useful, owing to its ability to quantify neuroaxonal loss [77], for phase II clinical trials of neuroprotective therapies. Over the last decade, OCT technology has expanded from a time-domain model to the more recent, spectral-domain model. Spectraldomain OCT is more rapid and with higher resolution $(5 \mu \mathrm{m}$ $v s 10 \mu \mathrm{m})$ than its predecessor [78]. The faster scanning speeds allow for much more information to be obtained in short periods of time, making it ideal for proof-of-concept studies [77]. OCT was initially used in patients with MS with optic neuritis, and RNFL has been shown to decrease by 10 to $40 \mu \mathrm{m}$ in the 3 to 6 months following an episode of acute optic neuritis [79]. Optic neuritis have been shown to involve not only inflammatory changes, but also neurodegeneration of optic nerve [80], and it has been proposed as a disease model to study potential neuroprotective and neurorestorative agents, with OCT measures of RNFL thickness and average macular thickness representing the primary outcome measures. A number of such trials have been or are currently being executed. In a phase II trial assessing erythropoietin in optic neuritis, RNFL quantification was used as the primary endpoint of the study [81]. In this study thickness of the RNFL decreased by a median of $7.5 \mu \mathrm{m}$ by week 16 versus a median of $16.0 \mu \mathrm{m}$ in the placebo group $(p=0.0357)$. These results give the first indications that erythropoietin might be neuroprotective in optic neuritis. Formal sample size calculation has been performed to assess the sample size needed for OCT as the primary outcome in optic neuritis studies [82]: for a 30\% reduction of the observed mean change of GCIP layer thinning from baseline to 6 months 159 patients per arm are needed for $90 \%$ power. No power calculations has been conducted yet in nonoptic neuritis MS, where larger numbers are expected. However, with newer techniques, more accurate segmentation and co-registration methods reduced variances and lower the sample sizes can be hypothesized [77]. 
It has been shown that RNFL and GCIP thinning is present even in the absence of a history of optic neuritis, demonstrating that there was ongoing axonal pathology even in the absence of an acute demyelinating event in the optic nerve [82]. In 2010, Talman et al. [83] showed progressive RNFL thinning by OCT over time in the absence of acute optic neuritis in a cohort of patients with MS studied longitudinally for up to 4 years of follow-up. This again reinforced the hypothesis that there is ongoing axonal degeneration in MS independent of acute inflammatory demyelinating disease. Recently effect of treatments emerged on OCT measures in patients with MS [84]: the effects of glatiramer acetate, natalizumab interferon- $\beta 1$ a subcutaneously and intramuscularly were assessed retrospectively on 402 patients. Rates of GCIP atrophy in this cohort of patients with RRMS vary according to DMT utilization, with lower GCIP thinning in patients treated with natalizumab. These findings support OCT for monitoring neurodegenerative treatment effects in the retina as a practical outcome measure in neuroprotection clinical trials.

While OCT can be used to obtain to a detailed characterization of retinal integrity, visual evoked potentials (VEP), however, allow us to characterize the functional integrity of the afferent visual pathway. In MS VEP usually present with an increased latency, which is thought to be a correlate of myelin loss, and often with reduced amplitude, which correlates with retinal neuroaxonal loss [85]. VEP can be used in association with OCT to identify those eyes with subclinical optic neuritis (which, in turn, could affect RNFL measures) [86]. Moreover, baseline VEP assessed together with other evoked potentials modalities (i.e., multimodal evoked potentials) have been shown to be associated with clinical disability worsening over 24 months in subjects with early RRMS [87]. The impact of DMT on evoked potentials, including VEP, has been evaluated in different studies and suggest their potential as an outcome marker in clinical trials. In a small study on clinically stable RRMS subjects treated with fingolimod, for example, an improvement in multimodal evoked potentials, including VEP, was observed after 1 year of treatment [88], whereas in an unblinded study of biotine VEP improvement was observed in a subgroup of patients [89]. VEP moreover, have been successfully used as an outcome markers of simvastatin treatment after acute optic neuritis [90]. Indeed, longitudinal VEP evaluation in MS subjects without previous optic neuritis and subclinical increased latencies has been proposed as a possible outcome for re-myelinating therapies trials [91]. However, preliminary work is needed in VEP protocol standardization between centers.

\section{Conclusions}

Here we have reviewed the more commonly available biomarkers that could play a role as outcome measures in phase
II clinical trials of DMT with tissue repair potential. We feel that to truly evaluate tissue repair it is important to differentiate it from other (anti-inflammatory and neuroprotective) possible therapeutic effects and thus to select biomarkers accordingly. Overall, phase II clinical trials of re-myelinating agents have already been completed or are well underway in MS [20], with changes in lesional MTR as the most frequently used MRI outcome measure [19]. While lesional MTR is far from ideal to evaluate remyelination in MS (as shown by the active research in qMTR and other markers), it is probably less than adequate to assess other facets of tissue repair such as neurogliogenesis and functional plasticity [92]. While functional and diffusion techniques both present with potential to tackle those other facets of tissue repair, more studies are needed to explore this. The time seems to be ripe for regenerative therapies to tackle MS. To this end, while development of powerful new DMT is key, more efforts seems to be needed also to hone imaging methods to capture adequately and reliably tissue repair.

\section{References}

1. Kutzelnigg A, Lassmann H. Pathology of multiple sclerosis and related inflammatory demyelinating diseases. Handb Clin Neurol 2014;122:15-58.

2. Colman D, Lubetzki C, Reingold S. Multiple paths towards repair in multiple sclerosis. Trends Neurosci 2003;26(2):59-61.

3. Miller DH, Grossman RI, Reingold SC, McFarland HF. The role of magnetic resonance techniques in understanding and managing multiple sclerosis. Brain 1998;121:3-24.

4. Vavasour IM, Li DK, Laule C, Traboulsee AL, Moore GR, Mackay AL. Multi-parametric MR assessment of T(1) black holes in multiple sclerosis : evidence that myelin loss is not greater in hypointense versus isointense T(1) lesions. J Neurol 2007;254(12):1653-1659.

5. Barkhof F, Bruck W, De Groot CJ, et al. Remyelinated lesions in multiple sclerosis: magnetic resonance image appearance. Arch Neurol 2003;60(8):1073-1081.

6. van Walderveen MA, Kamphorst W, Scheltens P, et al. Histopathologic correlate of hypointense lesions on T1weighted spin-echo MRI in multiple sclerosis. Neurology 1998;50(5):1282-1288.

7. Meier DS, Weiner HL, Guttmann CR. Time-series modeling of multiple sclerosis disease activity: a promising window on disease progression and repair potential? Neurotherapeutics 2007;4(3):485-498.

8. Rovira A, Auger C, Alonso J. Magnetic resonance monitoring of lesion evolution in multiple sclerosis. Ther Adv Neurol Disord 2013;6(5):298-310.

9. Meier DS, Weiner HL, Guttmann CR. MR imaging intensity modeling of damage and repair in multiple sclerosis: relationship of short-term lesion recovery to progression and disability. AJNR Am J Neuroradiol 2007;28(10):1956-1963.

10. Miller DH, Soon D, Fernando KT, et al. MRI outcomes in a placebo-controlled trial of natalizumab in relapsing MS. Neurology 2007;68(17):1390-1401. 
11. Schmierer K, Scaravilli F, Altmann DR, Barker GJ, Miller DH. Magnetization transfer ratio and myelin in postmortem multiple sclerosis brain. Ann Neurol 2004;56(3):407-415.

12. van Waesberghe JH, Kamphorst W, De Groot CJ, et al. Axonal loss in multiple sclerosis lesions: magnetic resonance imaging insights into substrates of disability. Ann Neurol 1999;46(5):747-754.

13. Filippi M, Rocca MA, Martino G, Horsfield MA, Comi G. Magnetization transfer changes in the normal appearing white matter precede the appearance of enhancing lesions in patients with multiple sclerosis. Ann Neurol 1998;43(6):809-814.

14. Richert ND, Ostuni JL, Bash CN, Leist TP, McFarland HF, Frank $\mathrm{JA}$. Interferon beta- $1 \mathrm{~b}$ and intravenous methylprednisolone promote lesion recovery in multiple sclerosis. Mult Scler 2001;7(1): 49-58.

15. Filippi M, Rocca MA, Sormani MP, Pereira C, Comi G. Shortterm evolution of individual enhancing MS lesions studied with magnetization transfer imaging. Magn Reson Imaging 1999;17(7):979-984

16. Chen JT, Collins DL, Atkins HL, Freedman MS, Arnold DL, Canadian MSBMTSG. Magnetization transfer ratio evolution with demyelination and remyelination in multiple sclerosis lesions. Ann Neurol 2008;63(2):254-262.

17. Altmann DR, Button T, Schmierer K, et al. Sample sizes for lesion magnetisation transfer ratio outcomes in remyelination trials for multiple sclerosis. Mult Scler Relat Disord 2014;3(2): 237-243.

18. Mallik S, Samson RS, Wheeler-Kingshott CA, Miller DH. Imaging outcomes for trials of remyelination in multiple sclerosis. J Neurol Neurosurg Psychiatry 2014;85(12):1396-1404.

19. van den Elskamp IJ, Knol DL, Vrenken H, et al. Lesional magnetization transfer ratio: a feasible outcome for remyelinating treatment trials in multiple sclerosis. Mult Scler 2010;16(6):660-669.

20. Schwartzbach CJ, Grove RA, Brown R, Tompson D, Then Bergh F, Arnold DL. Lesion remyelinating activity of GSK239512 versus placebo in patients with relapsing-remitting multiple sclerosis: a randomised, single-blind, phase II study. J Neurol 2017;264(2): 304-315.

21. Levesque IR, Sled JG, Narayanan S, et al. Reproducibility of quantitative magnetization-transfer imaging parameters from repeated measurements. Magn Reson Med 2010;64(2):391-400.

22. Simon JH. Brain atrophy in multiple sclerosis: what we know and would like to know. Mult Scler 2006;12(6):679-687.

23. De Stefano N, Airas L, Grigoriadis N, et al. Clinical relevance of brain volume measures in multiple sclerosis. CNS Drugs 2014;28(2):147-156.

24. Zivadinov R, Jakimovski D, Gandhi S, et al. Clinical relevance of brain atrophy assessment in multiple sclerosis. Implications for its use in a clinical routine. Expert Rev Neurother 2016;16(7):777-793.

25. Wang C, Beadnall HN, Hatton SN, et al. Automated brain volumetrics in multiple sclerosis: a step closer to clinical application. J Neurol Neurosurg Psychiatry 2016;87(7):754-757.

26. Kappos L, De Stefano N, Freedman MS, et al. Inclusion of brain volume loss in a revised measure of 'no evidence of disease activity' (NEDA-4) in relapsing-remitting multiple sclerosis. Mult Scler 2016;22(10):1297-1305.

27. Anderson VM, Bartlett JW, Fox NC, Fisniku L, Miller DH. Detecting treatment effects on brain atrophy in relapsing remitting multiple sclerosis: sample size estimates. J Neurol 2007;254(11): 1588-1594.

28. Altmann DR, Jasperse B, Barkhof F, et al. Sample sizes for brain atrophy outcomes in trials for secondary progressive multiple sclerosis. Neurology 2009;72(7):595-601.

29. Toth E, Szabo N, Csete G, et al. Gray matter atrophy is primarily related to demyelination of lesions in multiple sclerosis: a diffusion tensor imaging MRI study. Front Neuroanat 2017;11:23.
30. Biberacher V, Boucard CC, Schmidt P, et al. Atrophy and structural variability of the upper cervical cord in early multiple sclerosis. Mult Scler 2015;21(7):875-884.

31. Kalkers NF, Barkhof F, Bergers E, van Schijndel R, Polman CH. The effect of the neuroprotective agent riluzole on MRI parameters in primary progressive multiple sclerosis: a pilot study. Mult Scler 2002;8(6):532-533.

32. Liptak Z, Berger AM, Sampat MP, et al. Medulla oblongata volume: a biomarker of spinal cord damage and disability in multiple sclerosis. AJNR Am J Neuroradiol 2008;29(8):1465-1470.

33. Liu Z, Yaldizli O, Pardini M, et al. Cervical cord area measurement using volumetric brain magnetic resonance imaging in multiple sclerosis. Mult Scler Relat Disord 2015;4(1):52-57.

34. Wattjes MP, Harzheim M, Lutterbey GG, et al. Does high field MRI allow an earlier diagnosis of multiple sclerosis? J Neurol 2008;255(8):1159-1163.

35. Vrenken H, Geurts JJ, Knol DL, et al. Normal-appearing white matter changes vary with distance to lesions in multiple sclerosis. AJNR Am J Neuroradiol 2006;27:2005-2011.

36. Liu Z, Pardini M, Yaldizli O, et al. Magnetization transfer ratio measures in normal-appearing white matter show periventricular gradient abnormalities in multiple sclerosis. Brain 2015;138(Pt 5): 1239-1246.

37. Pardini M, Sudre CH, Prados F, et al. Relationship of grey and white matter abnormalities with distance from the surface of the brain in multiple sclerosis. J Neurol Neurosurg Psychiatry 2016;87(11):1212-1217.

38. Brown JW, Pardini M, Brownlee WJ, et al. An abnormal periventricular magnetization transfer ratio gradient occurs early in multiple sclerosis. Brain 2017;140(Pt 2):387-398.

39. Evangelou N, Esiri MM, Smith S, Palace J, Matthews PM. Quantitative pathological evidence for axonal loss in normal appearing white matter in multiple sclerosis. Ann Neurol 2000;47(3):391-395.

40. Kutzelnigg A, Lucchinetti CF, Stadelmann C, et al. Cortical demyelination and diffuse white matter injury in multiple sclerosis. Brain 2005;128(Pt 11):2705-2712.

41. Moll NM, Rietsch AM, Thomas S, et al. Multiple sclerosis normalappearing white matter: pathology-imaging correlations. Ann Neurol 2011;70(5):764-773.

42. Song SK, Yoshino J, Le TQ, et al. Demyelination increases radial diffusivity in corpus callosum of mouse brain. Neuroimage 2005;26(1):132-140.

43. Schmierer K, Wheeler-Kingshott CA, Boulby PA, et al. Diffusion tensor imaging of post mortem multiple sclerosis brain. Neuroimage 2007;35(2):467-477.

44. Wang Y, Wang Q, Haldar JP, et al. Quantification of increased cellularity during inflammatory demyelination. Brain 2011;134(Pt 12):3590-3601.

45. Wheeler-Kingshott CA, Cercignani M. About "axial" and "radial" diffusivities. Magn Reson Med 2009;61(5):1255-1260.

46. Zhang H, Schneider T, Wheeler-Kingshott CA, Alexander DC. NODDI: practical in vivo neurite orientation dispersion and density imaging of the human brain. Neuroimage 2012;61(4):1000-1016.

47. Muller HP, Turner MR, Grosskreutz J, et al. A large-scale multicentre cerebral diffusion tensor imaging study in amyotrophic lateral sclerosis. J Neurol Neurosurg Psychiatry 2016;87(6):570-579.

48. Mesaros S, Rocca M, Sormani M, et al. Bimonthly assessment of magnetization transfer magnetic resonance imaging parameters in multiple sclerosis: a 14-month, multicentre, follow-up study. Mult Scler 2010;16(3):325-331.

49. Arnold DL, Gold R, Kappos L, et al. Magnetization transfer ratio in the delayed-release dimethyl fumarate DEFINE study. J Neurol 2014;261(12):2429-2437. 
50. Schiepers C, Van Hecke P, Vandenberghe R, et al. Positron emission tomography, magnetic resonance imaging and proton NMR spectroscopy of white matter in multiple sclerosis. Mult Scler 1997;3(1):8-17.

51. Stankoff B, Freeman L, Aigrot MS, et al. Imaging central nervous system myelin by positron emission tomography in multiple sclerosis using [methyl-(1)(1)C]-2-(4'-methylaminophenyl)- 6hydroxybenzothiazole. Ann Neurol 2011;69(4):673-680.

52. Bodini B, Veronese M, Garcia-Lorenzo D, et al. Dynamic imaging of individual remyelination profiles in multiple sclerosis. Ann Neurol 2016 Feb 18.

53. Matias-Guiu JA, Cabrera-Martin MN, Matias-Guiu J, et al. Amyloid PET imaging in multiple sclerosis: an (18)F-florbetaben study. BMC Neurol 2015;15:243.

54. Jezzard P, Buxton RB. The clinical potential of functional magnetic resonance imaging. J Magn Reson Imaging 2006;23(6):787-793.

55. Enzinger C, Pinter D, Rocca MA, et al. Longitudinal fMRI studies: Exploring brain plasticity and repair in MS. Mult Scler 2016;22(3): 269-278.

56. Bonzano L, Pardini M, Mancardi GL, Pizzorno M, Roccatagliata L. Structural connectivity influences brain activation during PVSAT in Multiple Sclerosis. Neuroimage 2009;44(1):9-15.

57. Rocca MA, Colombo B, Falini A, et al. Cortical adaptation in patients with MS: a cross-sectional functional MRI study of disease phenotypes. Lancet Neurol 2005;4(10):618-626.

58. Filippi M, Rocca MA, Falini A, et al. Correlations between structural CNS damage and functional MRI changes in primary progressive MS. Neuroimage 2002;15(3):537-546.

59. Pardini M, Bonzano L, Roccatagliata L, Mancardi GL, Bove M. The fatigue-motor performance paradox in multiple sclerosis. Sci Rep 2013;3:2001.

60. Bonzano L, Pardini M, Roccatagliata L, Mancardi GL, Bove M. How people with multiple sclerosis cope with a sustained finger motor task: a behavioural and fMRI study. Behav Brain Res 2017;325(Pt A):63-71

61. Tomassini V, Matthews PM, Thompson AJ, et al. Neuroplasticity and functional recovery in multiple sclerosis. Nat Rev Neurol 2012;8(11):635-646.

62. Gorgolewski KJ, Storkey AJ, Bastin ME, Whittle I, Pernet C. Single subject fMRI test-retest reliability metrics and confounding factors. Neuroimage 2013;69:231-243.

63. Bosnell R, Wegner C, Kincses ZT, et al. Reproducibility of fMRI in the clinical setting: implications for trial designs. Neuroimage 2008;42(2):603-610

64. Kelly C, Biswal BB, Craddock RC, Castellanos FX, Milham MP. Characterizing variation in the functional connectome: promise and pitfalls. Trends Cogn Sci 2012;16(3):181-188.

65. Parisi L, Rocca MA, Valsasina P, Panicari L, Mattioli F, Filippi M. Cognitive rehabilitation correlates with the functional connectivity of the anterior cingulate cortex in patients with multiple sclerosis. Brain Imaging Behav 2014;8(3):387-393.

66. Dichter GS, Gibbs D, Smoski MJ. A systematic review of relations between resting-state functional-MRI and treatment response in major depressive disorder. J Affect Disord 2015;172:8-17.

67. Moccia M, Ciccarelli O. Molecular and metabolic imaging in multiple sclerosis. Neuroimaging Clin N Am 2017;27(2):343-356.

68. Ge Y, Gonen O, Inglese M, Babb JS, Markowitz CE, Grossman RI. Neuronal cell injury precedes brain atrophy in multiple sclerosis. Neurology 2004;62(4):624-627.

69. Khan O, Seraji-Bozorgzad N, Bao F, et al. The relationship between brain MR spectroscopy and disability in multiple sclerosis: 20-year data from the U.S. glatiramer acetate extension study. J Neuroimaging 2017;27(1):97-106.

70. Pardini M, Botzkowski D, Muller S, et al. The association between retinal nerve fibre layer thickness and $\mathrm{N}$-acetyl aspartate levels in multiple sclerosis brain normal-appearing white matter: a longitudinal study using magnetic resonance spectroscopy and optical coherence tomography. Eur J Neurol 2016;23(12):1769-1774.

71. Azevedo CJ, Kornak J, Chu P, et al. In vivo evidence of glutamate toxicity in multiple sclerosis. Ann Neurol 2014;76(2):269-278.

72. Hackmack K, Weygandt M, Wuerfel J, et al. Can we overcome the 'clinico-radiological paradox' in multiple sclerosis? J Neurol 2012;259(10):2151-2160.

73. Matthews PM, Pioro E, Narayanan S, et al. Assessment of lesion pathology in multiple sclerosis using quantitative MRI morphometry and magnetic resonance spectroscopy. Brain 1996;119 (Pt 3): 715-722.

74. Pardini M, Yaldizli O, Sethi V, et al. Motor network efficiency and disability in multiple sclerosis. Neurology 2015;85(13):1115-1122.

75. Bommarito G, Bellini A, Pardini M, et al. Composite MRI measures and short-term disability in patients with clinically isolated syndrome suggestive of MS. Mult Scler 2017:1352458517704077.

76. Balcer LJ, Raynowska J, Nolan R, et al. Validity of low-contrast letter acuity as a visual performance outcome measure for multiple sclerosis. Mult Scler 2017;23(5):734-747.

77. Gordon-Lipkin E, Calabresi PA. Optical coherence tomography: a quantitative tool to measure neurodegeneration and facilitate testing of novel treatments for tissue protection in multiple sclerosis. J Neuroimmunol 2017;304:93-96.

78. Galetta KM, Calabresi PA, Frohman EM, Balcer LJ. Optical coherence tomography (OCT): imaging the visual pathway as a model for neurodegeneration. Neurotherapeutics 2011;8(1):117-132.

79. Costello F, Coupland S, Hodge W, et al. Quantifying axonal loss after optic neuritis with optical coherence tomography. Ann Neurol 2006;59(6):963-969.

80. Guan Y, Shindler KS, Tabuena P, Rostami AM. Retinal ganglion cell damage induced by spontaneous autoimmune optic neuritis in MOG-specific TCR transgenic mice. J Neuroimmunol 2006;178(12):40-48.

81. Suhs KW, Hein K, Sattler MB, et al. A randomized, double-blind, phase 2 study of erythropoietin in optic neuritis. Ann Neurol 2012;72(2):199-210.

82. Syc SB, Saidha S, Newsome SD, et al. Optical coherence tomography segmentation reveals ganglion cell layer pathology after optic neuritis. Brain 2012;135(Pt 2):521-533.

83. Talman LS, Bisker ER, Sackel DJ, et al. Longitudinal study of vision and retinal nerve fiber layer thickness in multiple sclerosis. Ann Neurol 2010;67(6):749-760.

84. Button J, Al-Louzi O, Lang A, et al. Disease-modifying therapies modulate retinal atrophy in multiple sclerosis: a retrospective study. Neurology 2017;88(6):525-532.

85. Thurtell MJ, Bala E, Yaniglos SS, Rucker JC, Peachey NS, Leigh RJ. Evaluation of optic neuropathy in multiple sclerosis using low-contrast visual evoked potentials. Neurology 2009;73(22):1849-1857.

86. Naismith RT, Tutlam NT, Xu J, et al. Optical coherence tomography is less sensitive than visual evoked potentials in optic neuritis. Neurology 2009;73(1):46-52.

87. Jung P, Beyerle A, Ziemann U. Multimodal evoked potentials measure and predict disability progression in early relapsing-remitting multiple sclerosis. Mult Scler 2008;14(4):553-556.

88. Iodice R, Carotenuto A, Dubbioso R, Cerillo I, Santoro L, Manganelli F. Multimodal evoked potentials follow up in multiple sclerosis patients under fingolimod therapy. J Neurol Sci 2016;365: 143-146.

89. Sedel F, Papeix C, Bellanger A, et al. High doses of biotin in chronic progressive multiple sclerosis: a pilot study. Mult Scler Relat Disord 2015;4(2):159-169.

90. Tsakiri A, Kallenbach K, Fuglo D, Wanscher B, Larsson H, Frederiksen J. Simvastatin improves final visual outcome in acute optic neuritis: a randomized study. Mult Scler 2012;18(1):72-81. 
91. Niklas A, Sebraoui H, Hess E, Wagner A, Then Bergh F. Outcome measures for trials of remyelinating agents in multiple sclerosis: retrospective longitudinal analysis of visual evoked potential latency. Mult Scler 2009;15(1):68-74.
92. Pluchino S, Zanotti L, Brini E, Ferrari S, Martino G. Regeneration and repair in multiple sclerosis: the role of cell transplantation. Neurosci Lett 2009;456(3):101-106. 